\title{
Five regions, five retinopathy screening programmes: a systematic review of how Portugal addresses the challenge
}

\author{
Andreia Marisa Penso Pereira ${ }^{1 *}$, Raul Manuel da Silva Laureano ${ }^{2^{*}}$ and Fernando Buarque de Lima Neto ${ }^{3}$
}

\begin{abstract}
Background: The implementation of a population-based screening programme for diabetic retinopathy involves several challenges, often leading to postponements and setbacks at high human and material costs. Thus, it is of the utmost importance to promote the sharing of experiences, successes, and difficulties. However, factors such as the existence of regional programmes, specificities of each country's health systems, organisational and even linguistic barriers, make it difficult to create a solid framework that can be used as a basis for future projects.
\end{abstract}

Methods: Web of Science and PubMed platforms were searched using appropriate key words. The review process resulted in 423 articles adherent to the search criteria, 28 of which were accepted and analysed. Web sites of all Portuguese governmental and non-governmental organisations, with a relevant role on the research topic, were inspected and 75 official documents were retrieved and analysed.

Results: Since 2001, five regional screening programmes were gradually implemented under the guidelines of Portuguese General Health Department. However, complete population coverage was still not achieved. Among the main difficulties reported are the complex articulation between different levels of care providers, the low number of orthoptic technician in the national health system, the high burden that images grading, and treatment of positive cases represents for hospitals ophthalmology services, and low adherence rates. Yet, the comparison between strategies adopted in the different regions allowed the identification of potential solutions: hire orthoptic technician for primary health care units, eliminating the dependence of hospital professionals; use artificial intelligence algorithms for automatic retinographies grading, avoiding ophthalmologists overload; adoption of proximity strategies, as the use of portable retinographers, to promote adherence to screening.

Conclusion: Access to diabetic retinopathy screening remains remarkably variable in Portugal and needs urgent attention. However, several characteristics of effective screening programmes were found in Portuguese screening programmes, what seems to point toward promising outcomes, especially if each other highlights are considered. The findings of this research could be very useful for the other countries with similar socio-political characteristics.

Trial registration: PROSPERO registration ID CRD42020200115.

Keywords: Diabetic retinopathy, Population-based screening, Systematic review, Portuguese screenings

\footnotetext{
*Correspondence: andreia_marisa_pereira@iscte-iul.pt; raul.laureano@iscte-

iul.pt

'Instituto Universitário de Lisboa (ISCTE-IUL), ISTAR-IUL, Av. das Forças

Armadas, 1649-026 Lisbon, Portugal

${ }^{2}$ Instituto Universitário de Lisboa (ISCTE-IUL), Business Research Unit (BRU-IUL) and ISTAR-IUL, Av. das Forças Armadas, 1649-026 Lisbon, Portugal

Full list of author information is available at the end of the article
}

(c) The Author(s). 2021 Open Access This article is licensed under a Creative Commons Attribution 4.0 International License, which permits use, sharing, adaptation, distribution and reproduction in any medium or format, as long as you give appropriate credit to the original author(s) and the source, provide a link to the Creative Commons licence, and indicate if changes were made. The images or other third party material in this article are included in the article's Creative Commons licence, unless indicated otherwise in a credit line to the material. If material is not included in the article's Creative Commons licence and your intended use is not permitted by statutory regulation or exceeds the permitted use, you will need to obtain permission directly from the copyright holder. To view a copy of this licence, visit http://creativecommons.org/licenses/by/4.0/ The Creative Commons Public Domain Dedication waiver (http://creativecommons.org/publicdomain/zero/1.0/) applies to the data made available in this article, unless otherwise stated in a credit line to the data. 


\section{Contributions}

- This study contributes to the assemblage of knowledge in the field of diabetic retinopathy screenings, providing the first systematic review of the Portuguese experience.

- The study also details the main diabetic retinopathy screening implementation problems. It points out the possible solutions for operational planning of future screenings, the improvement possible for the existing ones, and put forward a framework to comparative analyses.

- This study highlights the importance of adequate governmental funding, national guidelines that precise the role of the different intervenient, and of politic measures that guarantee the involvement of all parts.

\section{Background}

Diabetes Mellitus (DM) is a chronic metabolic disease and one of the most prevalent diseases worldwide [1-4]. DM can cause macro and microvascular complications, including diabetic retinopathy (DR) [5-7]. DR occurs when blood vessels in the light-sensitive region of the eye, the retina, leak or become blocked, due to prolonged high blood glucose levels $[8,9]$. DR is the most common cause of vision loss in people with diabetes $[7$, $10]$ and globally is the leading cause of visual impairment and blindness among working age population [1113]. However, DR can be prevented or delayed by timely diagnosis and management of diabetes [14, 15], and blindness can also be prevented or delayed by regular eye screening and appropriate treatment $[16,17]$.

Nonetheless, although extremely important, the implementation of a population-based DR screening, requires the intervention of many stakeholders (government, hospitals, primary health care units) and involves numerous challenges, which often lead to unexpected setbacks at high human and material costs $[18,19]$. Thus, the share of knowledge and experiences between countries is of recognised utility, and there is a permeant need for a solid framework, that can be used as a basis for future projects $[5,18,20]$. However, the desirable interchange is not easy to accomplish. In fact, different countries often have different health systems, which makes it difficult to understand and categorise procedures $[5,21]$, screening programmes may be implemented at a national, regional, or local level, resulting in sparse information at a national level, and there are organisational and even linguistic barriers, that complicate the process $[18,20]$.

In this context, this study intends to answer the following research question: How is the population-based DR screening programme conducted in Portugal? And, consequently, to contribute to the assemblage of knowledge in the field of DR screening, providing a systematic scientific and technical literature review of the Portuguese experience, which can be used to plan future programmes or implement improvements in the existing ones.

The strategic planning of a DR screening requires a deep knowledge to be successful [20]. So, in this paper five key questions are addressed, namely: i) What are the general guidelines of the screening programmes in Portugal? ii) How did each region implement the screening? iii) What are the main metrics used to measure the results of each screening programme and how did DR Screening results evolved through time? iv) What are the main problems reported when implementing DR screening programmes and how can eventual risks be mitigated?

By analysing the accepted 28 scientific peer-reviewed articles and 75 technical documents from government (e.g., [22, 23]) and non-governmental organisations (e.g., $[24,25])$, five Portuguese regional DR screening programmes, within the context of the National Health System (SNS), allowed the identification of the advantages and weaknesses of each regional strategy and are discussed in the light of documented international experiences. Most of the available studies about DR screening are cost-effectiveness analyses (e.g., [26, 27]), or are focused on very specific aspects of the process (for example automatic reading grading $[19,28])$. However, the overall screening strategy is rarely well described [16] and normally only the unilateral point of view of one type of stakeholder is explored, e.g., diabetics [29], health professionals [30], primary health care units [30], hospitals [31] and government [27]. As opposed to that, in this review we specifically tried to identify alternative screening strategies and assess the challenges faced by the different levels of health care providers, producing a synthesis of the evidence available in the literature.

This work is organised as follows. The first section concerns the adopted methodology and literature selection. Then, the general guidelines of the screening programme in Portugal, the differences between regional protocols, the indicators used to measure screening results, the quality evaluation, and the main problems reported in implementing DR screening programmes, are analysed. Finally, the implications of the different scenarios are examined considering the best national and international practices.

\section{Methods}

\section{Search for studies}

We performed a systematic review according to the Preferred Reporting Items for Systematic Reviews and Meta-Analysis (PRISMA) checklist [32] (see Additional file 1). 
For the scientific review, Web of Sciences (www. webofknowledge.com) and PubMed (https://www.ncbi. nlm.nih.gov/pubmed/) databases were searched. The selection of scientific databases was based on their scope and their wide range of publications in the field of interest [33, 34]. Moreover, these databases are frequently used in other researches [33].

The search was performed according to the following query: (("Diabetic retinopathy" or "DR" or "diabetic vision lost" or "diabetic complication") and ("screening" or "preventive public policy" or "preventive eye exam" or "early diagnosis" or "retinography") and ("population based" or "mass")).

The query was applied to the topic (title, abstract and keywords) field, for the period 2009-2020 and only considering articles written in English or Portuguese languages. The time constraint was imposed because, in Portugal, there is no truly population-based DR screening, prior to the year 2009. The linguistic restriction is due to the very purpose of this systematic review - to analyse the screening of DR in Portugal - and, to the fact, that English is nowadays the universal language in the scientific world.

Technical documents were retrieved from the web sites of all Portuguese governmental and nongovernmental organisations, with a relevant role on DR Screening (see Additional file 2). Governmental organisations were selected based on their mission and in the organisational chart of the National Health System. Non-governmental organisations were identified through references of papers and official documents.

\section{Inclusion and exclusion criteria}

Inclusion and exclusion criteria were applied to select the relevant set of articles to be reviewed.

For the scientific review, were included studies published in pear review journals, referring to the DR screening programme in Portugal. Articles that were focused on interventions, clinical rehearsals, research with methodological deficiencies, and duplicate work were excluded. Four hundred and twenty-three articles were retrieved from Web of Sciences and PubMed databases. A preliminary review process was applied according to the following steps: 1) exclusion duplicate articles; 2) evaluation of scientific articles according to abstracts excluding those focused-on interventions, clinical rehearsals, research with methodological deficiencies. This preliminary evaluation resulted on the exclusion of 64 articles.

For the technical review were included only official documents, available on the institution web site, and referring to DR screening programme. Excluded documents were those that are not dully substantiated and duplicate work. Regarding technical documents, 1 hundred and 75 were retrieved from the web sites of all
Portuguese governmental and non-governmental organisations (listed in Additional file 2), with a relevant role on DR Screening. After the preliminary evaluation, 97 official documents were selected and analysed.

Finally, all the selected documents were submitted to a critical full document evaluation, what allowed to exclude articles that did not mentioned the Portuguese DR screening programmes, scientific or technical documents with methodological deficiencies and the ones not dully substantiated. Two experts of the Portuguese North Region Health Administration validated both selection procedures. After the selection process, 28 articles and 25 official documents remained. Figure 1 illustrates the selection process.

\section{Articles and documents analyses procedure}

To facilitate the documents analysis, they were organised in different categories. The documents classification was carried out by two of the three authors of this systematic review. The third researcher was called to break the tie, whenever there was no agreement between the first two.

Scientific documents were divided in three categories: i) DR Incidence / prevalence studies or studies focus on DR characteristics, such as risk factors, natural history and, progression (10 papers); ii) Machine learning algorithms for images grading (10 papers); and iii) Screening strategies (five papers). Three papers were classified in both 1 and 2 categories. Additionally, to access the quality of the scientific articles eight quality items were considered (Table 1) and graded according with the following rule: $\operatorname{Yes}(\mathrm{Y})=1 ; \operatorname{No}(\mathrm{N})=0 ; \operatorname{Partially}(\mathrm{P})=0.5$. The marking of the selected papers in each of the quality criteria is available in Additional file 3.

Official technical documents were classified as documents of national scope (24) or documents of regional scope (51). Regional documents were distributed by North (11), Central (10), Lisbon and Tagus Valley (10), Alentejo (10) and Algarve (10) regions.

\section{Results \\ General guidelines of the Portuguese screening programme}

In 1998, the Portuguese General Health Department (DGS) has established the first guidelines for DR population-based screening programmes. Non-mydriatic Chamber Fundus Photography (colour retinography) was the recommended screening method, due to its high sensitivity and specificity (92 and 90\% respectively), and because this method can be performed by trained paramedical personnel and later sent for ophthalmologist analyses. Annual screenings were recommended for diabetics after puberty [22]. The costs of the screening and treatment for DR are completely covered by the government. Only indirect costs, as transportation to the 


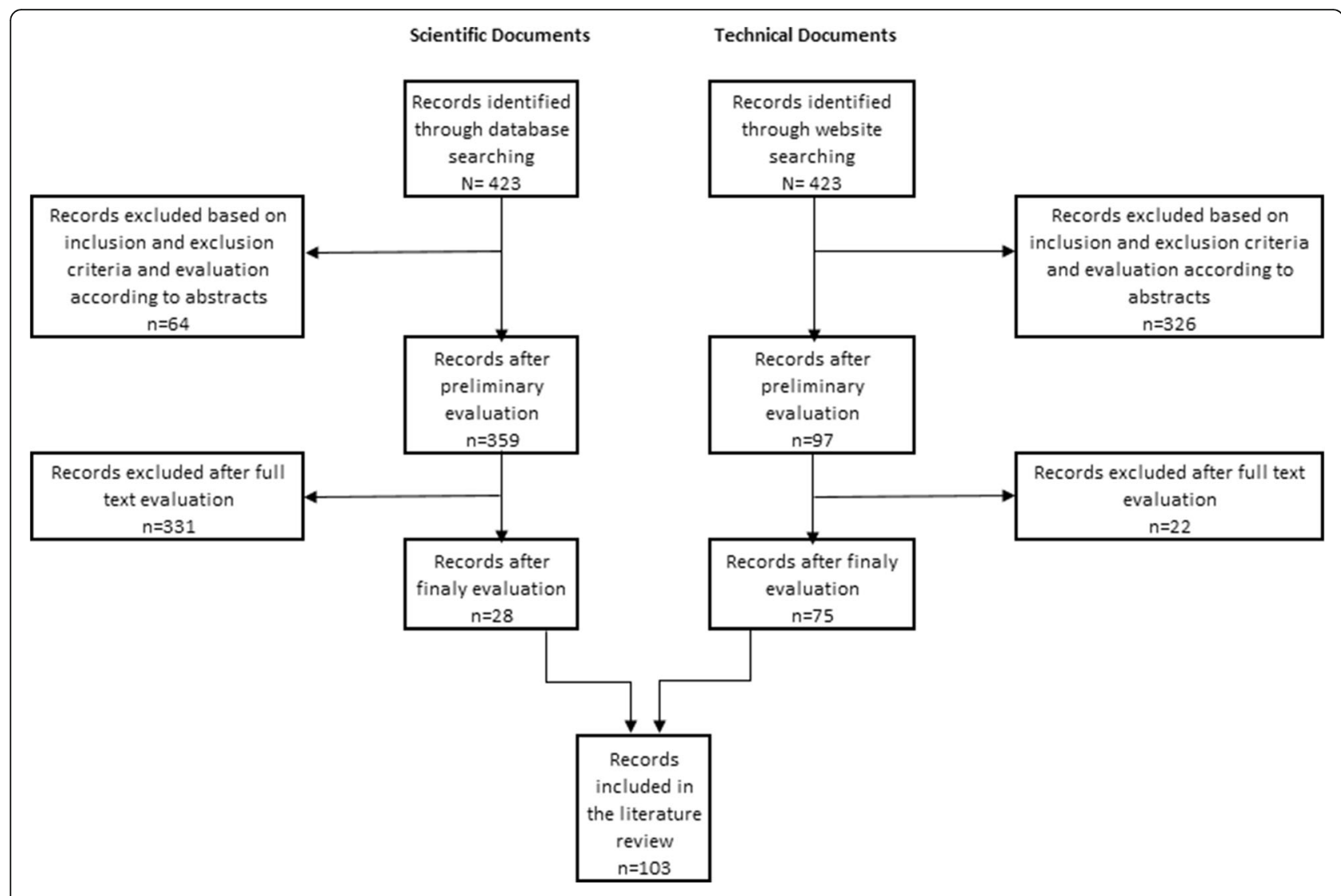

Fig. 1 Flow chart of scientific/technical documents selection process

screening or treatment facility, are supported by the diabetics [22]. Regional Health Administrations (ARS) have the responsibility of operationalise population-based screening programmes. In Portugal there are five ARS (ARS North, Central, Lisbon and Tagus Valley, Alentejo and Algarve). So, since 2001, the ARS began the implementation of screening strategies under DGS guidelines [35-39]. None the less, the guidelines were vague in what concerns to major operationalisation aspects as what services and health staff should be involved and which are their responsibilities, where the screening test should take place, who identifies and convokes the diabetic populations, etc. Therefore, the strategies adopted by each ARS are significantly different [35-39]. Regarding positive cases, all the ARS mention referral for a hospital ophthalmology consultation, where a diagnosis is made and a treatment plan appropriate to the stage of the disease is established. However, despite the treatment being guaranteed, there were no guidelines for its standardisation at national level. The definition of a

Table 1 Quality Criteria Items

\begin{tabular}{ll}
\hline ID & Quality Criteria \\
\hline PQ02 & Are the details of the screening protocol well described? \\
PQ03 & Are the sources reliable? \\
PQ04 & Is the methodology used rigorous and replicable? \\
PQ03 & The geographical area covered and the institution responsible for the screening are well identified? \\
PQ04 & Are the indicators used to measure screening results well described? \\
PQ05 & Does it identify the problems that affect the implementation of population-based screening programs? \\
PQ06 & Does it identify the constraints that affect the implementation of population-based screening programs? \\
PQ07 & Does it identify solutions and best practices from national or international experiences concerning population-based DR screening? \\
PQ08 & Does it objectively describe the evolution of DR screening over a considerably large period? \\
\hline
\end{tabular}


positive case itself, that is, requiring referral for ophthalmology consultation, was not uniform in all regions [35-39].

In 2018, DGS issued new and more detailed, guidelines for the organisation of regional screening programmes [40], proposing a flow chart for the screening process (Fig. 2).

DR Grading and the definition of Positive and Negative, were also clarified and normalised, trough the referral guidelines summarised in Table 2 [40].

Table 3 summarises the DGS recommended procedures and treatments for the different stages of DR [40].

\section{Regional DR screening protocols}

At the North Regional Health Administration (ARSN), the DR Screening Programme began in 2009, and has been gradually implemented in the following years. In 2009, ARSN, developed exhaustive proceedings, documentation, and protocols, which have been subsequently expanded and adjusted [39]. In this region, retinographies are performed in Primary Health Centres. However, there are no fixed retinographers in health facilities. The equipment remains in mobile units, moving from health centre to health centre, according to prior established schedule [39]. Primary Health Centres are responsible for identifying and convening the diabetic population and retinographies are performed by orthoptics technicians. However, there are no orthoptics technicians dedicated solely to the screening programme. Those professionals are provided by local hospitals, and usually accumulate the functions inherent to the screening programme, with the functions they perform regularly in the hospitals. After the retinographies are performed they are analysed and graded by ophthalmologists [39]. ARSN is conducting a research aiming the introduction of automatic image reading software in DR screening programme, however, this technology is still experimental [42]. After the grading, positive cases are referred to the hospital for treatment. Since the beginning of the screening programme, ARSN uses the International Clinical Classification System, which categorises DR severity in 5 levels, including 3 stages of low risk: none, mild, and moderate NPDR, a fourth stage of severe NPDR, and a fifth stage of PDR, in the presence or absence of DME, which is graded separately (as recommended by the 2018 DGS guidelines - Table 1) $[39,40]$. The ARSN uses a specific software to support the screening programme (SIIMAScreenings) [23].

At the Portuguese Central Region Health Administration (ARSC), the DR Screening Programme is running since 2001 [37]. As in the North Region, the screening method and the target population follow the 1998 DGS guidelines [37]. Until 2011, the screening protocol was similar to the one implemented at ARSN. However, in that year, was introduced the use of an automatic image reading software (RetmarkerSR) in conjunction with the traditional human analysis and grading. This software allows the detection of RD lesions such as DME and small haemorrhages in retinal photographs, through a method based on image processing algorithms [37]. Two of the selected papers focus on the performance of this particular software revealing a sensitivity of $99.76 \%$ and a specificity of $99.49 \%$ [43, 44]. The grading scale used in ARS Centro, is different from the 2018 DGS guidelines. The scale includes 5 different classification levels: NC - not classifiable; R0 - no DR lesions; RL - NPDR without maculopathy; M - maculopathy; and RP - PDR. In ARS Centro, referable diabetic retinopathy, was defined for all patients graded as NPDR, PDR, or M [43]. Another particularity of ARSC Screening is that there is no software application to support the screening programme. The data are requested by the ARSC to each of the Primary Health Centre Clusters (ACES) and compiled into Excel sheets [37].

Lisbon and Tagus Valley Regional Health Administration (ARSLVT) and the Association for the Protection of Diabetics of Portugal (APDP) signed a cooperation protocol in 2009, for DR screening [38]. It was the beginning of Diabetic Retinopathy Screening Service for Lisbon and Tagus Valley (RETINODIAB), commissioned and driven by APDP and supported by ARSLVT. The RETINODIAB follows the 1998 DGS norms in terms of screening test and target population [45]. In 2016, the ARSLVT implemented their own pilot screening in four ACES. Accordingly, with this established protocol, the retinographies are performed by orthoptists, in the ACES, and automatically analysed and graded by a software - "Retmarker". When classified by the software as "necessary human reading", they are sent for ophthalmologists' analysis. The results of these readings are made available to the family doctor by means of a computerised screening platform. As in ARSN, the DR grading scale used is according to the 2018 DGS guidelines [45]. Positive (except Mild NPDR) and inconclusive cases are referenced to hospital ophthalmology services [38]. Nowadays, ARSLVT, extended this new screening programme, and APDP, RETINODIAB, is still a complementary response, continuing to cover 7 of the 15 ACES [38]. In ARSLVT, the screening programme is computer-supported by SIIMAScreenings in 4 ACES and by the APDP system in 7 [38]. An internal recruitment process for orthoptists for Primary Health Care has begun in 2017 [38].

At Alentejo Regional Health Administration (ARS Alentejo), there is no standardised screening strategy. In fact, there are three different screenings. The DR screening managed by ARS Alentejo, which began in 2011 and follows the 1998 DGS guidelines in terms of method and 


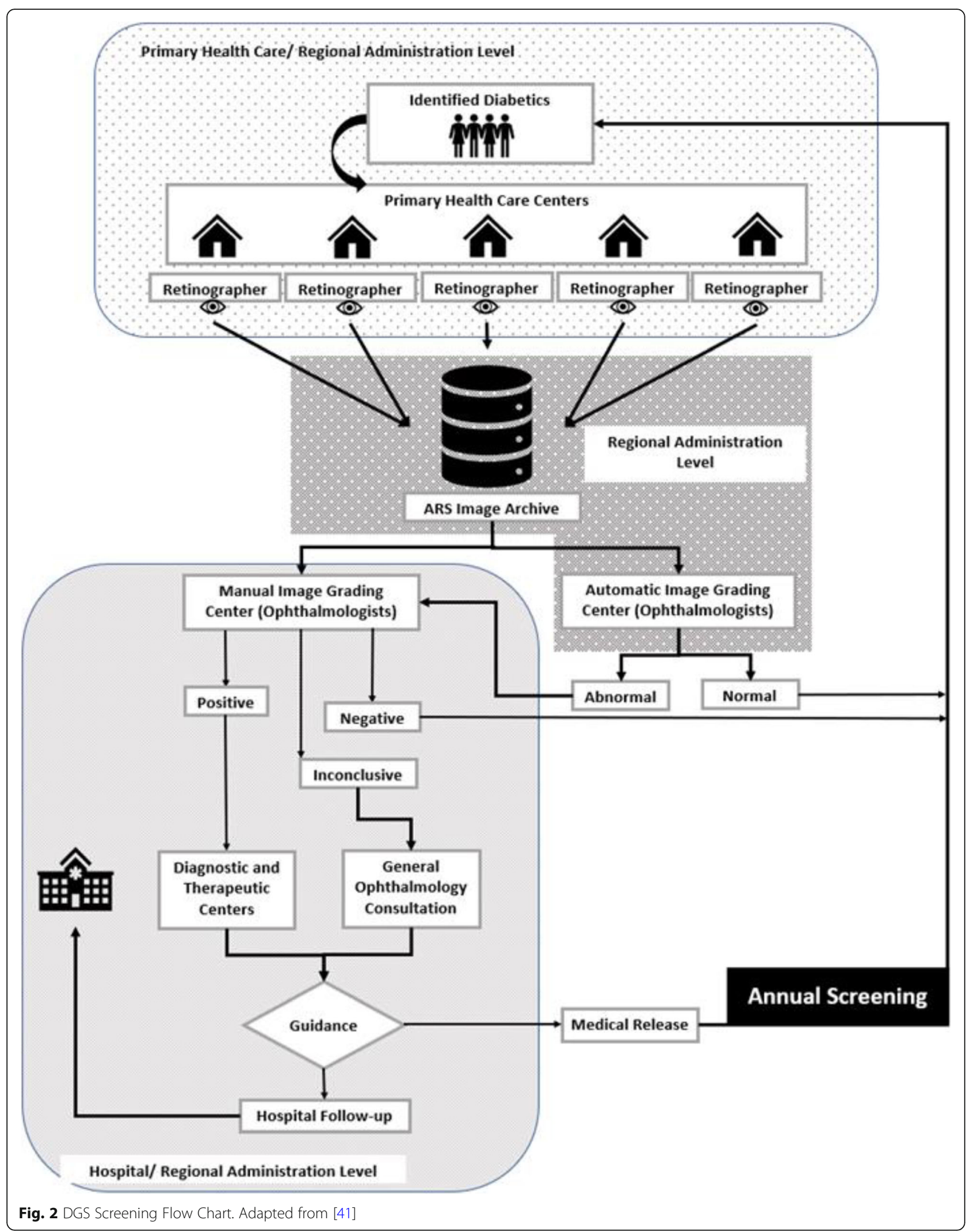


Table 2 DGS 2018 referral guidelines. Adapted from [40]

\begin{tabular}{lll}
\hline \multicolumn{2}{l}{ Diabetic retinopathy screening result } & Referral \\
R0 & No disease visible & Repeats screening after a year \\
R1 & Mild No Proliferative DR (NPDR) & Repeats screening after a year \\
R2 & Moderate NPDR & CDTI 1, 2, 3 RD ophthalmologic consultation in a two-month period \\
R3 & Severe NPDR & CDTI 2, 3 RD ophthalmologic consultation in 1 month period \\
& $\begin{array}{l}\text { Proliferative DR (PDR) } \\
\text { M1 } \quad \text { Diabetic Macular Edema (DME) }\end{array}$ \\
V1 $\quad$ Hight risk PDR, vitreous haemorrhage, or tractional retinal detachment & CDTI 3 RD ophthalmologic consultation in a 15-day period \\
ICN $\quad$ Inconclusive or comorbidities & General ophthalmology consultation \\
Treatment follow-uo & \\
P0 $\quad$ Stable LASER & Repeats after a year \\
P1 $\quad$ Insufficient LASER & CDTI 1 (Thermic LASER) \\
\hline
\end{tabular}

target population, is implemented in one ACES. The retinographies are performed by orthoptic technicians provided by hospitals and uses SIIMAScreenings as screening computer-system [35]. In a second ACES, family doctors refer patients with diabetes to perform the retinography in the hospital, so the data related to this ACES are not introduced in the screening platform. And, in a third area the screening is carried out in partnership with APDP [35].

In March 2013, the Algarve Regional Health Administration (ARS Algarve) began the implementation of a populationbased screening for all diabetics in the region [36].

The screening test is performed by the two Hospitals in Algarve, in the ophthalmology departments. The articulation between ARS Algarve and the hospitals is performed through protocols and annual contracting. Screening monitoring is computer-supported [36].

During the year 2014, hospitals were reticent about the renewal of the screening protocol due to the reduce installed capacity. So, ARS Algarve proposed to limit the screening, in this period, to the "new cases" diagnosed during 2013 and 2014 what was accomplished by the end of the year [36]. In
2015 and 2016, the screening was resumed in a normal way. However, in 2017 and 2018, the screening did not take place. In that year's activities report, ARS Algarve claims that, although the normal procedures for the renewal of the programme were carried out, there was any hospital response and that, despite having taken countless efforts to develop a screening programme less dependent on hospital capacity (similar to those existing in the North, Centre and part of Lisbon and Vale do Tejo), this was not possible due to numerous procedural constraints [36].

The analysis of the technical documentation of the five ARS, showed that there are considerable differences between the implemented screening programmes (Table 4) [35-39]:

- The screening location varies according to the region: in ARSN and ARSC there are portable retinographers which, in turn, are allocated to the Primary Health Centres of the region [37, 39]; at ARSLVT there are fixed retinographers in Primary Health Care units [38], and in ARS Algarve all screening phases are performed by hospital ophthalmology services.

Table 3 DGS 2018 treatment guidelines. Adapted from [40]

\begin{tabular}{|c|c|}
\hline Diabetic retinopathy stage & Procedure and treatment \\
\hline No disease visible or Mild NPDR & DR screening \\
\hline NPDR moderate or severe & $\begin{array}{l}\text { DR ophthalmologic consultation } \\
\text { Optical coherence tomography (OCT) }\end{array}$ \\
\hline $\begin{array}{l}\text { NPDR with DME focal or multifocal or PDR } \\
\text { without DME }\end{array}$ & $\begin{array}{l}\text { Fluorescein angiography (FA) and OCT } \\
\text { Laser therapy }\end{array}$ \\
\hline $\begin{array}{l}\text { PDR with MDE } \\
\text { Diffuse DME }\end{array}$ & $\begin{array}{l}\text { FA + OCT } \\
\text { Combined DR therapy: Laser + Intravitreal injection of anti-vascular endothelial growth and/or long- } \\
\text { acting corticosteroids }\end{array}$ \\
\hline $\begin{array}{l}\text { Advanced PDR with: } \\
\text { - Vitreous or sub hyaloid haemorrhage } \\
\text { - Retinal detachment } \\
\text { - Neovascular glaucoma } \\
\text { - Chronic DME with no response to } \\
\text { treatment or refractory }\end{array}$ & $\begin{array}{l}\text { DR chirurgic therapy: vitrectomy } \\
\text { Combined DR therapy: FA + OCT + Laser + Intravitreal injection of anti-vascular endothelial growth } \\
\text { and/or long-acting corticosteroids } \\
\text { Corticosteroids extended-release injectable devices }\end{array}$ \\
\hline
\end{tabular}


Table 4 Screening Protocol

\begin{tabular}{|c|c|c|c|c|c|}
\hline & North & Central & LVT & Alentejo & Algarve \\
\hline $\begin{array}{l}\text { Screening } \\
\text { method - colour } \\
\text { fundus } \\
\text { photography }\end{array}$ & Yes & Yes & Yes & Yes & Yes \\
\hline $\begin{array}{l}\text { Electronic } \\
\text { transfer of } \\
\text { images }\end{array}$ & Yes & Yes & Yes & Yes & Yes \\
\hline $\begin{array}{l}\text { Retinografers } \\
\text { location }\end{array}$ & Portable & Portable s & Non-portable - ACES & $U$ & Hospital \\
\hline Pupil dilatation & No & No & No & No & No \\
\hline $\begin{array}{l}\text { Calls to the } \\
\text { target population } \\
\text { through postal } \\
\text { invitations }\end{array}$ & Yes & Yes & Yes & Yes & Yes \\
\hline $\begin{array}{l}\text { Who performs } \\
\text { the photography }\end{array}$ & $\begin{array}{l}\text { Orthoptic technicians } \\
\text { provided by hospitals }\end{array}$ & $\begin{array}{l}\text { Orthoptic technicians provided by } \\
\text { hospitals }\end{array}$ & $\begin{array}{l}\text { Primary Health Centres } \\
\text { orthoptists }\end{array}$ & $U$ & $\begin{array}{l}\text { Hospital } \\
\text { Orthoptic } \\
\text { technicians }\end{array}$ \\
\hline $\begin{array}{l}\text { Software for } \\
\text { automatic } \\
\text { readings }\end{array}$ & No & Yes & Yes & No & No \\
\hline Camara Device & $\begin{array}{l}\text { Non-mydriatic camera, CR-2 } \\
\text { Digital Retinal Camera (Canon) }\end{array}$ & $\begin{array}{l}\text { Nonmydriatic cameras - Canon CR6- } \\
45 \mathrm{NM} \text { with a Sony DXC-950P 3CCD } \\
\text { colour video camera }\end{array}$ & $\begin{array}{l}\text { Non-mydriatic camera, CR-2 } \\
\text { Digital Retinal Camera (Canon) }\end{array}$ & $U$ & $U$ \\
\hline $\begin{array}{l}\text { Screening test } \\
\text { procedure }\end{array}$ & $\begin{array}{l}\text { Retinography of both retinal } \\
\text { fields, both with } 45^{\circ} \text { field, one } \\
\text { focusing on the macula and } \\
\text { the other on the optic nerve }\end{array}$ & $\begin{array}{l}\text { Retinography of both retinal fields, } \\
\text { both with } 45^{\circ} \text { field, one focusing on } \\
\text { the macula and the other on the optic } \\
\text { nerve. When impossible to obtain an } \\
\text { image with minimum quality is } \\
\text { performed an iatrogenic pupil dilation } \\
\text { with a topical mydriatic. }\end{array}$ & $\begin{array}{l}\text { Retinography of both retinal } \\
\text { fields, both with } 45^{\circ} \text { field, one } \\
\text { focusing on the macula and } \\
\text { the other on the optic nerve }\end{array}$ & $U$ & $U$ \\
\hline
\end{tabular}

U Unavailable

- If, in some ARS, retinographies are performed by hospital orthoptic technicians, which accumulate the functions in the hospital with the DR screening [39], other (ARSLVT) are hiring optometrists for primary health care units [38]. Although this solution seems simple and effective on eliminating the dependence of available hospital technicians, it is not easy to implement, mostly due to the lack of consensus on the competence of optometrists to perform retinographies. In fact, there are substantial differences in the training of the two types of professionals: orthoptic technicians are qualified to detect vision abnormalities and ocular motility disorders. Therefore, the orthoptic technician is active in diagnosis, therapy, and rehabilitation; on the other hand, optometrists are the professionals that, through examination of the eye, diagnoses refractive errors and prescribes appropriate lenses and/or exercises, without the need for drug or surgical treatments [40, 46]. However, there are several countries in which the retinographies are carried out by professionals other than orthotics technicians or optometrists, for example, primary care physicians or nurses [17, 47], but in Portugal those options were never considered.
- In the ARSC and in part of the ARLVT region, artificial intelligence software is implemented for automatic retinographies grading [43-45]. Several studies state its acceptable sensitivity and specificity levels and its effectiveness to reduce ophthalmology services burden [48-50].

The new DGS directives substantiate an important attempt to guarantee quality, equity of access and standardisation of screening at national level [40]. However, the analysis of the latest activity reports of the ARS (2018), clearly shows that, so far, the new guidelines have not produced many effects at the regional level. Thus, while some ARS established procedures perfectly framed with the guidelines now issued, there are others, in which the socalled population-based screening programmes fall far short of the requirements that the denomination, and the current national guidelines, require [35-39].

\section{Main indicators and screening results}

The analysis of the official reports of the Portuguese institutions directly involved in the implementation of the DR screenings allowed to determine a set of common 
indicators, used to monitor the process and the results of the screening programmes.

However, the number of available indicators is very small, reflecting only the concern with the coverage of the screening [35-39]. No indicators inherent to the quality of the process were found in any of the five ARS. In rare cases, references to the evolution of the number of positive DR cases were found, which, however, were discarded due to important inconsistencies in the concept of "positive case" itself. Still, it was found that most ARS collect and report the following indicators [35-39]:

$$
\begin{aligned}
& \text { Geographic coverage }=\frac{\text { Number of ACES on the programme }}{\text { Total ACES of the Region }} \\
& \text { Adherence rate }=\frac{\text { Number of retinographies }}{\text { Number of invitations }} \\
& \text { Population coverge }=\frac{\text { Number of invitations }}{\text { Number of identified diabetics }} \\
& \text { Screened population }=\frac{\text { Number of retinographies }}{\text { Number of identified diabetics }}
\end{aligned}
$$

As previously mentioned, generally, the indicators are calculated by the ARS, although the data are obtained directly through an operating system dedicated to screening, or indirectly, through requests to the primary health units, or associations involved (APDP, hospitals) Of course, when the second case occurs, less reliability of the data is expected, since it is common for different entities to follow different criteria for extracting and pre-processing the information.

But, in addition to this issue, there are other inconsistencies in the calculation of the indicators [35-39]:

1- First, as we have seen, there are several ARSs (part of ARS LVT, ARS Alentejo and ARS Algarve) where screening is still conducted, in whole or in part, by other institutions, leaving the question of whether it is truly a population-based screening. Normally, the ACES where this happens are counted as being covered by a screening programme, but, at the risk of, in some cases, providing only an opportunistic screening to registered diabetics. This inconsistence will affect the "Geographic Coverage" indicator.

2- The variable "number of identified diabetics" is also likely to introduce some bias in the analysis of the results. In reality, not all identified diabetics are convolved into screening. According to the DGS guidelines, family doctors should remove from the list the subjects who are unable to remain seated, those who underwent a retinography less than a year ago and those who are blind. Thus, it is important to distinguish whether the ARS account for the initial number of identified diabetics, or that obtained after the purging of the initial listings. The "Population coverage" and "Screened population" indicators could be affected by these decisions.

3- The variable "Number of invitations" is also not easy to measure. In fact, so far, none of the ARS has managed to strictly comply with the 12-month interval between screenings. Therefore, at the time of the change of civil year, there are several locations with the annual screening still in progress. Thus, these questions arise: is it effective only to consider invitation letters in places where the screening has already been completed? All invitation letters sent should be considered, even if, in some cases diabetics have not yet had the opportunity to adhere to the screening, simply because, the screening was scheduled for a date later than the present moment? The assumptions in each case are not clear and may condition the comparison of adherence rates between ARS. The "Population coverage" and the "Adherence rate" are affected by this bias.

Despite the constraints mentioned previously, the following Tables 5, 6, and 7 show the available indicators, in each of the five ARS. Due to the scarcity of information in some of the ARS, it was decided to present the results only for 2015 and 2017 (years in which more comprehensive information was obtained) [39]. The variable "Number of retinographs performed" was the only one that allowed an evolutionary analysis, which is presented in Table 7 [35-39].

Despite several setbacks in all regions, the number of screenings has been increasing since 2009. In 2015, a total of 113,443 retinographies were taken, $19 \%$ more than in the same period of 2014 (Table 7). However, access to diabetic retinopathy screening is still remarkably variable in Portugal and needs urgent attention. Population coverage, in 2017 varies from 0\% in ARS Algarve to $100 \%$ in ARSLVT (Table 6) [35-39].

\section{Discussion}

Retinopathy screening involves several interfaces where communication can be problematic (family doctor, patients, optometrists, regional screening teams, hospitals, ophthalmologists) $[20,21,51,52]$. A major effort is necessary to understand and coordinate this complex system with dynamic interactions of different agents (stakeholders, providers, professionals, and individuals), and where change in any one element can alter the context for all other elements [20]. So, national guidelines should precise the role of the different intervenient, and politic measures should be created to guarantee the involvement of all parts. 
Table 52015 Results

\begin{tabular}{|c|c|c|c|c|c|c|}
\hline & North & Central & LVT & Alentejo & Algarve & Total \\
\hline ACES on the programme (a) & 17 & 8 & 11 & 4 & 2 & 42 \\
\hline Total ACES (b) & 24 & 8 & 15 & 4 & 3 & 54 \\
\hline Geographic coverage (a)/(b) & $70.8 \%$ & $100.0 \%$ & $73.3 \%$ & $100.0 \%$ & $66.7 \%$ & $77.8 \%$ \\
\hline Identified diabetics (c) & 277,706 & 142,008 & 183,958 & 47,221 & $U$ & 674,537 \\
\hline Number of invitations (d) & 75,767 & $U$ & 57,049 & 3501 & 23,404 & 159,721 \\
\hline Number of retinographies (e) & 45,119 & 19,792 & 35,602 & 3477 & 16,491 & 120,481 \\
\hline Percentage of ungradable images & $3,2 \%$ & $3,5 \%$ & $3,7 \%$ & $U$ & $U$ & $U$ \\
\hline Adherence rate $=(e) /(d)$ & $59.5 \%$ & $U$ & $62.4 \%$ & $99.3 \%$ & $70.5 \%$ & $75.4 \%$ \\
\hline Population coverage $=(d) /(c)$ & $27.3 \%$ & $U$ & $31,0 \%$ & $7,4 \%$ & $U$ & $23.7 \%$ \\
\hline Screened population $=(e) /(c)$ & $16.2 \%$ & $13.9 \%$ & $19.4 \%$ & $7.4 \%$ & $U$ & $17.9 \%$ \\
\hline
\end{tabular}

U Unavailable

According to official documents, another of the major problems for the sustainability of Portuguese screening programmes is the lack of orthoptic technicians in the SNS. ARS where retinographies are performed by hospital orthoptic technicians, which accumulate the functions in the hospital with the DR screening, are dealing with permanent difficulties to ensure the full coverage of the programme $[37,39]$. In fact, this situation led to interruption of screenings in sites that had already started and can represent a major sustainability problem [39]. In addition, some ARS reported difficulties in ensuring the first hospital visit within 30 days of the diagnosis of DR [39].

To truly understand these problems is important to know the hospitals point of view. Opportunely, one of the selected studies took place at the Hospital Centre of Oporto (CHP), and provides the perspective of this hospital ophthalmology services [31]. The CHP is the reference hospital for 2 ARSN's ACES, which together represent about 293,900 inhabitants and 24,902 diabetics (data for 2016) [31]. An important finding that emerges from this research is that the screening programme is referencing to ophthalmologic consultation, patients who are already being followed in hospital services. ARSN screening protocol recommends the exclusion of these cases from the call lists [23], however, hospitals and primary health care computer systems are not fully integrated, and family physicians do not always have access to information to identify those situations. During the period under review, $56 \%$ of referrals were cancelled due to this reason [31]. The same study also refers to the overloading of ophthalmology services with the dispensing of orthoptic technicians for screening. The authors conclude that, the screening programme relevance and advantage to public health is evident. However, they highlight that at a time when involvement in the programme represents an increased effort for ophthalmology services, it is important to optimise all steps of the process [31].

Hire orthoptic technicians exclusively for the screening programme could lighten the effort of hospital services, however, for that to happened, it is necessary to ensure an increased number of university positions in courses for orthoptic technicians [38]. On the other hand, optometrists claim for a more relevant role in DR screening planning and implementation [46]. In this context, ARS LTV is already hiring optometrists for primary health care units [38]. In England, this solution is implemented in a broader way. There are some regions where retinographies are carried out at high-street optometrists with cooperation protocols [17, 30, 47]. However, studies show that, in some of those areas, there are problems with access due to long waiting lists. So, uptake rates have not been found to be higher for those accessing screening services via high-street optometrists, despite this modality of screening being thought to offer increased proximity to the patients and appointment flexibility $[18,19,29]$. In Spain and in Mexico,

Table 62017 Results

\begin{tabular}{llllll}
\hline & North & Central & LVT & Alentejo & Algarve \\
\hline Geographic coverage 2017 & $75 \%$ & $63 \%$ & $100 \%$ & $50 \%$ & $0 \%$ \\
Adherence rate 2017 & $60 \%$ & $U$ & $52 \%$ & $91 \%$ & $\mathrm{NA}$ \\
Screened population $\mathbf{2 0 1 7}$ & $35 \%$ & $9 \%$ & $29 \%$ & $6 \%$ & 0 \\
\hline
\end{tabular}

U Unavailable, NA Not Applied 
Table 7 Evolution of the number of retinographies

\begin{tabular}{llllllllll}
\hline ARS & $\mathbf{2 0 0 9}$ & $\mathbf{2 0 1 0}$ & $\mathbf{2 0 1 1}$ & $\mathbf{2 0 1 2}$ & $\mathbf{2 0 1 3}$ & $\mathbf{2 0 1 4}$ & $\mathbf{2 0 1 5}$ & $\mathbf{2 0 1 6}$ & $\mathbf{2 0 1 7}$ \\
\hline North & 791 & 8839 & 39,006 & 49,354 & 57,385 & 47,454 & 45,121 & 68,309 & 105,462 \\
Central & 14,760 & 15,271 & 15,258 & 18,496 & 11,856 & 13,235 & 19,792 & $\cup$ & $U$ \\
LTV & 3131 & 13,867 & 23,221 & 24,819 & 28,272 & 25,853 & 28,562 & 35,602 & 74,744 \\
Alentejo & $\mathrm{U}$ & 2761 & 2872 & 2512 & 1668 & 7573 & 3477 & 7144 & 2799 \\
Algarve & 10,907 & 9395 & 13,580 & 7937 & 16,103 & 1420 & 14,491 & $U$ & 0 \\
\hline
\end{tabular}

U Unavailable

retinographies and the first interpretation of the test are performed by family nurses or physicians. Then, there is a second valuation by the ophthalmologist, who knows the previous diagnosis and sends his opinion to primary care [17].

Computer systems are also important in the screening process: maintaining and sharing disease registers across different agents, management of patient records; automatic call/recall routines, electronic image transfer, and programme monitoring, are some aspects where new technologies have a critic role $[16,47]$.

The other major problem reported by CHP is the high burden that image grading and treatment of positive cases represents for hospitals ophthalmology services [31, 51]. Portugal may have about 1 million people with diabetes, of whom 700,000 diagnosed and on medical treatment and who should be consulted annually according to the DGS criteria. According to the Portuguese Ophthalmology Society, each of the 988 Portuguese ophthalmologists (2014 data) would have to observe about 708/each year, an infeasible number in terms of logistics specialty requirements. Moreover, only 422 of the 988 Portuguese ophthalmologists works in the SNS [53]. Automated grading software can decrease the cost of screening and reduce the amount of work for retinal grading ophthalmologists $[8,19,28,52]$. Studies suggest it has an acceptable level of accuracy $[19,43,44,48,49]$, and, besides the two Portuguese regions (ARSC and ARSLVT), it is already implemented throughout Scotland, in parts of Spain, Denmark and Hungary $[16,47,50]$.

Mobile units using non-mydriatic cameras, may have an important role in increasing rates of screening attendance [47], another of the constrains mentioned by ARS.

In most ARS it has not been possible to have an annual frequency of generalised screening [51]. The implementation of screening programmes with extended intervals (more than 12 months between tests) may, in fact, be an option to free up resources and provide better care, but there are some concerns around this subject [20]. Actually, there are three determining factors when considering the use of extended screening intervals: the control of the diabetes, the sensitivity of the screening test and the adherence rate [20]. If the first two are objective and easy to quantify, the third factor may have more complex implications. Even if the rate of adherence of a certain population is high, it is possible that by increasing the interval between screenings, the message that the test is not important is being involuntarily transmitted, which can in the medium-term lead to a decrease of population adherence and consequently making the use of extended intervals a risky option [20]. Currently in Europe, the implementation of screening programmes with intervals of more than 1 year between calls is already quite frequent. However, some countries have adopted this measure in conjunction with the increased frequency of screening for diabetics identified as high risk (usually with calls every 6 months) $[11,47,50]$. On the other hand, the results of these options are not completely consistent. In Denmark and Finland there are no reports of problems associated with the increase of screening intervals, while in Sweden, the adherence rate has dropped significantly after the adoption of this measure (although the cause-and-effect relationship has not been fully proven) [47].

The actual practice in other countries shows that the medium- and long-term effect of rigorous screening implementation is effective [21, 47]. The United Kingdom began in the 1960s to screen diabetic retinopathy nationally and transversally [24]. It is concluded that in the 2009-10 biennium, for the first time, diabetic retinopathy was not the first cause for the attestation of incapacity for visual blindness of working age in England and Wales, 40 years after the implementation of the screening [24, 47]. Therefore, it is not expected that the implementation of public policies on diabetes and diabetic retinopathy lead to visible results in 3 or 4 years, but those results should appear in the medium and long term [21].

\section{Conclusions}

This study allowed the analysis of the diabetic retinopathy screenings implemented in mainland Portugal. There was some difficulty in collecting uniform data since there are different degrees of implementation, methodologies, and monitoring in the five ARS. However, this analysis allows to assess the differences, detect constraints, and identify possible solutions and improvements. 
The main conclusion is that access to diabetic retinopathy screening remains remarkably variable in Portugal and needs urgent attention. Due to its importance DR screening should be a public health priority, and governments should ensure adequate funding to populationbased programmes. National guidelines should also precise the role of the different intervenient, and politic measures should be created to guarantee the involvement of all parts.

Even though characteristics of effective screening programmes (adequate sensitivity and specificity, a convenient method for the patient, proximity strategies) were found in Portuguese screening programmes, which could be pointing towards promising outcomes, we notice lots of room for improvement. With a continued effort, hopefully, in a few years there will be a national, standardised, population-based, DR screening programme.

The findings of this research could be very useful for other Countries with similar socio-political characteristics.

\section{Abbreviations \\ ACES: Primary Health Centre Clusters; APDP: Association for the Protection of Diabetics of Portugal; ARS: Regional Health Administrations; ARS Alentejo: Alentejo Regional Health Administration; ARS Algarve: Algarve Regional Health Administration; ARSC: Central Region Health Administration; ARSLVT: Lisbon and Tagus Valley Regional Health Administration; \\ ARSN: North Regional Health Administration; CHP: Hospital Centre of Oporto; DGS: Portuguese General Health Department; DM: Diabetes Mellitus; DR: Diabetic Retinopathy; PRISMA: Preferred Reporting Items for Systematic Reviews and Meta-Analysis; SNS: National Health System; RETINODIAB: Diabetic Retinopathy Screening Service for Lisbon and Tagus Valley}

\section{Supplementary Information}

The online version contains supplementary material available at https://doi. org/10.1186/s12913-021-06776-8.

Additional file 1. PRISMA 2009 Checklist.

Additional file 2. Overview of the Portuguese governmental and nongovernmental health organizations, with a relevant role on DR Screening. Additional file 3. Quality assessment of the selected scientific papers.

\section{Acknowledgements}

We are grateful to the Physicians of North Regional Health Administration who provided insight and expertise that greatly assisted this systematic review.

\section{Authors' contributions}

$A P, R L$ and $F N$ author conceived the design of the study. AP and $R L$ led the review of abstracts, titles, and full texts, with support from FN for data abstraction. AP led the drafting of the manuscript, with all authors contributing to review, feedback, and collective decision-making. The author(s) read and approved the final manuscript.

\section{Funding}

This work is partially funded by national funds through FCT - Fundação para a Ciência e Tecnologia, I.P., under the projects UID/GES/00315/2020 and UIDB/04466/2020, and by Iscte under the project HOPE.

\section{Availability of data and materials}

Abstracted data collected and analysed during this study and described in this systematic review will be available from the corresponding author upon request.

\section{Declarations}

Ethics approval and consent to participate

This systematic review was conducted using published data, and study authors were contacted for additional contextual information, thus, the need for written ethics approval and consent to participate is not applicable.

\section{Consent for publication}

Not applicable.

\section{Competing interests}

The authors declare that they have no competing interests.

\section{Author details}

'Instituto Universitário de Lisboa (ISCTE-IUL), ISTAR-IUL, Av. das Forças Armadas, 1649-026 Lisbon, Portugal. ${ }^{2}$ Instituto Universitário de Lisboa (ISCTE-IUL), Business Research Unit (BRU-IUL) and ISTAR-IUL, Av. das Forças Armadas, 1649-026 Lisbon, Portugal. ${ }^{3}$ Escola Politécnica, Computer Engineering (POLI/PPG-EC), Universidade de Pernambuco (UPE), Rua Benfica, 455 - Bloco 'C', Recife 50720-001, Brazil.

Received: 6 May 2021 Accepted: 21 July 2021

Published online: 30 July 2021

\section{References}

1. Williams R, Airey M, Baxter H, Forrester J, Kennedy-Martin T, Girach A. Epidemiology of diabetic retinopathy and macular edema: a systematic review. Eye. 2004;18(10):963-83. https://doi.org/10.1038/sj.eye.6701476.

2. Wild S, Roglic G, Green A, Sicree R, King H. Global prevalence of diabetes: estimates for the year 2000 and projections for 2030. Diabetes Care. 2004; 27(5):1047-53. https://doi.org/10.2337/diacare.27.5.1047.

3. Beagley, Guariguata L, Weil C, Motala A. Global estimates of undiagnosed diabetes in adults. Diabetes Res Clin Pract. 2014;IO3:150-60.

4. Tamayo T, Rosenbauer J, Wild SH, Spijkerman A, Baan C, Forouhi N, et al. Diabetes in Europe: an update. Diabetes Res Clin Pract. 2014;103:206-17.

5. Chen E, Looman M, Laouri M, Gallagher M. Burden of illness of diabetic macular edema: Literature review. Curr Med Res Opin. 2010;26(7):1587-97.

6. Xiao D, Bhuiyan A, Frost S, Vignarajan, Mei-Ling, Tay-Kearney, et al. Major automatic diabetic retinopathy screening systems and related core algorithms: a review. Machine Vision Appl. 2019;30(3):423-46.

7. Gadkari SS, Maskati B, Nay BK. Prevalence of diabetic retinopathy in India: the all India ophthalmological society diabetic retinopathy eye screening study 2014. Indian J Ophthalmol. 2016;64(1):38-44. https://doi.org/10.4103/ 0301-4738.178144.

8. Goh JKH, Cheung CY, Sim SS, Tan PC, Tan SW, Wong TY. Retinal imaging techniques for diabetic retinopathy screening. J Diabetes Sci Technol. 2016; 10(2):282-94. https://doi.org/10.1177/1932296816629491.

9. Tracey ML, Gilmartin M, O'Nei K, Fitzgerald AP, McHugh SM, Buckley CM, et al. Epidemiology of diabetes and complications among adults in the Republic of Ireland 1998-2015: a systematic review and meta-analysis. BMC Public Health. 2016;16:132.

10. Lee, Wong TY, Saba C. Epidemiology of diabetic retinopathy, diabetic macular edema and related vision loss. Eye Vision. 2015;2:17.

11. Echouffo-Tcheugui JB, Ali MK, Roglic, Hayward RA, Narayan KM. Screening intervals for diabetic retinopathy and incidence of visual loss: a systematic review. Diabetic Med. 2013;30:1272-92.

12. Lin YT, Chen YC, Peng YT, Chen L, Liu JH, Chen FL, et al. Evidence-based medicine of screening of diabetic retinopathy among type 2 diabetes: a clinical overview. Health. 2015;7(07):873-83. https://doi.org/10.4236/health.2 015.77103.

13. Sivaprasad S, Gupta B, Crosby-Nwaobi R, Evans J. Prevalence of diabetic retinopathy in various ethnic groups: a worldwide perspective. Survey Ophthalmol. 2012;57: № 4.

14. World Health Organization. Diabetes. Available from: https://www.who.int news-room/fact-sheets/detail/diabetes. Accessed 20 July 2020. 
15. Elwali ES, Almobarak AO, Hassan MA, Mahmooud AA, Awadalla H, Ahmed $\mathrm{MH}$. Frequency of diabetic retinopathy and associated risk factors in Khartoum, Sudan: population based study. Int J Ophthalmol. 2017;10(6):94854. https://doi.org/10.18240/ijo.2017.06.18.

16. European Conference on Screening for Diabetic Retinopathy. Screening for diabetic retinopathy in Europe, Moving forward from the St Vincent Declaration. Liverpool: Royal Liverpool University Hospital; 2005.

17. Pieczynski J, Grzybowski. Review of diabetic retinopathy screening methods and Programmes adopted in different parts of the world. Eur Ophthal Rev. 2015;9(1):49-55. https://doi.org/10.17925/EOR.2015.09.01.49.

18. World Health Organization/ International Foundation Europe. The Saint Vicent Declaration on diabetes care and research in Europe. In Diabet Med. 1990;7(4):360.

19. Roy R, Lob A, Pal BP, Oliveira CM, Raman R, Shar T. Automated diabetic retinopathy imaging in Indian eyes: A pilot study. Indian J Ophthalmol. 2015;62: $\mathrm{N}^{\circ} 2$.

20. Ting DSW, Chui, Wong TY. Diabetic retinopathy: global prevalence, major risk factors, screening practices and public health challenges: a review. Clin Exp Ophthalmol. 2016;44(4):260-77. https://doi.org/10.1111/ceo.12696.

21. Scanlon P. Diabetic retinopathy screening: Progress or lack of Progress. In: Visual Dysfunction in Diabetes. Part of the Ophthalmology Research book series (OPHRES). New York; Springer; 2012. p. 17-29.

22. General Health Department. Circular Normativa N: 7/DGCG. Lisbon: Diagnóstico Sistemático e Tratamento da Retinopatia; 1998.

23. North Regional Health Administration. Procedures manual for diabetic Retinophaty screening in Northen region. Porto: North Regional Health Administration; 2009

24. International Diabetes Federation. Diabetes atlas. Brussels: International Diabetes Federation; 2015.

25. Portuguese Diabetes Association - APDP. Home page. Available from: https://www.apdp.pt/. Accessed 13 Feb 2020.

26. Pasquel, Hendrick A, Ryan M, Cason E, Ali M, Narayan KMV. Costeffectiveness of different diabetic retinopathy screening modalities. J Diabetes Sci Technol. 2016;10(2):301-7. https://doi.org/10.1177/193229681 5624109.

27. Byrne, Parker DF, Tannenbaum SL, Ocasio M, Lam B, Zimmer-Galler, et al. Cost of a Community-Based Diabetic Retinopathy Screening Program. Diabetes Care. 2014;37:236-7

28. Hansen MB, Tang HL, Wang S, Turk LA, Piermarocchi R, Speckauskas M, et al. Automated detection of diabetic retinopathy in three European populations. J Clin Exp Ophthalmol. 2016;7:4.

29. Trento M, Bajardi, Borgo E, Passera P, Maurino M, Gibbins R, et al. Perceptions of diabetic retinopathy and screening procedures among diabetic people. Diabet Med. 2002;19(10):810-3. https://doi.org/10.1046/j.14 64-5491.2002.00784.x.

30. Lindenmeyer A, Sturt J, Hipwell A, Stratton I, Al-Athamneh N, Gadsby R, et al. Influence of primary care practices on patients' uptake of diabetic retinopathy screening: a qualitative case study. Br J Gen Pract. 2014;64(625): e484-92. https://doi.org/10.3399/bjgp14X680965.

31. Abreu AC, Lages $V$, Batista $P$, Ribeiro A, Menéres $P$, Pessoa B. First 5 years of implementation of diabetic screening program in Centro Hospitalar do Porto. Rev Bras Oftalmol. 2017;76(6):295-9.

32. Moher D. LATJ. Preferred reporting items for systematic reviews and metaanalyses: the PRISMA statement. Ann Intern Med. 2009;151:264-9.

33. Falagas ME, Pitsouni El, Malietzis GA, Pappas G. Comparison of PubMed, Scopus, Web of Science, and Google Scholar: strengths and weaknesses. FASEB J. 2007:22(2):338-42.

34. Gasparyan AY. Bibliographic databases: some critical points. Korean Acad Med Sci. 2013;28(6):799-800. https://doi.org/10.3346/jkms.2013.28.6.799.

35. Alentejo Regional Health Administration. Annual activities report 2008-2017. Alentejo Regional Health Administration, Évora, Portugal, 2009-2018. Avaiable from: http://www.arsalentejo.min-saude.pt/arsalentejo/ InstrumentosGestao/Paginas/Instrumentos-de-Gest\%C3\%A3o.a spx?RootFolder=\%2Farsalentejo\%2FInstrumentosGestao\%2FDocuments\%2 FRelat\%C3\%B3rio\%20de\%20Atividades\&FolderCTID=0x012000E3CD169AB4 081943A3CCC71554ED99CD\&View $=\{95599779-F E A 6-468 E-B F 2 C-31 D 94 C 8 B 4$ $8 \mathrm{~F} 2$.

36. Algarve Regional Health Administration. Annual activities report 2007-2018 Algarve Regional Health Administration, Faro, 2008-2019. Avaiable from: http://www.arsalgarve.min-saude.pt/instrumentos-de-gestao-2/.
37. Central Regional Health Administration. Annual activities report 2006-2017. Central Regional Health Administration, Coimbra, 2007-2018. Avaiable from: http://www.arscentro.min-saude.pt/instrumentos-de-gestao/relatorios-de-a tividades/\#content.

38. Lisbon and Tagus Valley Regional Health Administration. Annual activities report 2007-2017. Lisbon and Tagus Valley Regional Health Administration, Lisbon, 2008-2019. Avaiable from: http://www.arslvt.min-saude.pt/ instrumentos-de-gestao/relatorios-de-atividades/\#content.

39. North Regional Health Administration. Annual activities report 2007-2017. North Regional Health Administration, Porto 2008-2018. Avaiable from: http://www.arsnorte.min-saude.pt/instrumentos-de-gestao-2/relatorio-de-a ctividades/\#content.

40. General Health Department. Norma 016/2018 - Rastreio da Retinopatia Diabética. 2018.

41. General Health Department. Available from: https://www.dgs.pt/. Accessed 13 Feb 2019.

42. Pedrosa M, Silva J, Silva J, Matos S, Costa C. SCREEN-DR: collaborative platform for diabetic retinopathy. Int J Med Inform. 2018;120:137-46. https://doi.org/10.1016/j.ijmedinf.2018.10.005.

43. Ribeiro L, Oliveira C, Neves C, Ramos J, Ferreira H, Cunha-Vaz J. Screening for Diabetic Retinopathy in the Central Region of Portugal. Added Value of Automated 'Disease/No Disease' Grading. Ophthalmologica. 2014;233:96103.

44. Oliveira C, Cristovão L, Ribeiro ML, Abreu J. Improved automated screening of diabetic retinopathy. Ophthalmologica. 2011;226(4):191-7. https://doi. org/10.1159/000330285.

45. Medeiros M, Mesquita E, Papoila A, Genro V, Raposo J. First diabetic retinopathy prevalence study in Portugal: RETINODIAB study-evaluation of the screening programme for Lisbon and Tagus Valley region. $\mathrm{Br} \mathrm{J}$ Ophthalmol. 2015;99(10):1328-33.

46. Portuguese Optometrist Association - APOR. Available from: https://www.a por-ortoptistas.com.pt/?p=areasactuacao_ortoptica. Accessed 15 Jan 2020.

47. European Association for the Study of Diabetes. Screening for Diabetic Retinopathy in Europe -Progress Since 2011. Manchester: 26th European Association for the Study of Diabetes Eye Complications Study Group, University of Manchester; 2016.

48. Nørgaard M, Grauslund J. Automated Screening for Diabetic Retinopathy A Systematic Review. Ophthal Res. 2018.

49. Valverde C, Garcia M, Hornero R, Lopez-Galvez MI. Automated detection of diabetic retinopathy in retinal images. Indian J Ophthalmol. 2016;64(1):2632. https://doi.org/10.4103/0301-4738.178140.

50. Sim DA, Keane PA, Aiello LP, Silva PS. Automated retinal image analysis for diabetic retinopathy in telemedicine. Curr Diabetes Rep. 2015;15(3):14. https://doi.org/10.1007/s11892-015-0577-6.

51. Rodrigues J. Implementação do rastreio da retinopatia diabética: Relatório de estudo de caso Porto: Rede do Centro Hospitalar do Porto; 2014.

52. Almdal T, Handlos L, Valerius M, Juul E, Nielsen K, Vistisen D, et al. Glycaemic threshold for diabetes-specific retinopathy among individuals from Saudi Arabia, Algeria and Portugal. Diabetes Res Clin Pract. 2014;103:e44-6.

53. Central Administration of the Health System - ACSS. Rede Nacional de Especialidade Hospitalar e de Referenciação: Oftalmologia. 2017.

\section{Publisher's Note}

Springer Nature remains neutral with regard to jurisdictional claims in published maps and institutional affiliations.

\section{Ready to submit your research? Choose BMC and benefit from:}

- fast, convenient online submission

- thorough peer review by experienced researchers in your field

- rapid publication on acceptance

- support for research data, including large and complex data types

- gold Open Access which fosters wider collaboration and increased citations

- maximum visibility for your research: over $100 \mathrm{M}$ website views per year

At BMC, research is always in progress.

Learn more biomedcentral.com/submission 